\title{
Lösung komplexer Optionsbewertungsprobleme mittels stochastischer Simulation und dynamischer Programmierung
}

\author{
Dr. Oliver Mußhoff und Dr. Norbert Hirschauer, Berlin
}

Es ist bekannt, dass analytische Lösungsverfahren bzw. die Binomialmethode bei schwierigen Optionsbewertungsproblemen nicht gangbar oder zumindest sehr aufwändig sind. In diesem Beitrag wird eine effiziente und gleichzeitig intuitive Vorgehensweise skizziert, die durch die Kombination von rückwärts-rekursiver Programmierung und stochastischer Simulation eine genaue Bewertung amerikanischer Optionen mit geringem Aufwand ermöglicht.

Dr. Oliver Mußhoff und Dr. Norbert Hirschauer forschen und lehren als Wissenschaftliche Mitarbeiter am Fachgebiet Allgemeine Betriebslehre des Landbaus an der Humboldt-Universität zu Berlin mit den Arbeitsschwerpunkten Investition, Finanzierung, Risikomanagement und Qualitätssicherung.

Oliver Mußhoff dankt der Klauis-Tschira-Stiftung, gemeinnütze GmbH, für die finanzielle Unterstützung.

\section{Optionen: Struktur und Bewertungs- verfahren}

Optionen erfreuen sich als Spekulations- und Absicherungsinstrument einer immer größer werdenden Beliebtheit. So werden bspw. Optionen auf Aktien, Indizes, Währungen, Zinstitel und Rohstoffe (z. B. Kupfer, Rohöl und landwirtschaftliche Produkte) angeboten. Ganz allgemein berechtigen Optionen den Inhaber dazu, einen Vermögenswert (Underlying) mit unsicherer zukünftiger Wertentwicklung zu einem vorab vereinbarten Preis zu kaufen bzw. zu verkaufen. Die Wahrnehmung dieses Rechts ist i.d.R. zu mehreren Zeitpunkten innerhalb eines festgelegten Zeitraumes möglich (amerikanische Optionen). Nicht zuletzt seit der nobelpreisgekürten Entwicklung der modernen Optionspreistheorie durch Black, Scholes und Merton ist die Bestimmung des Wertes von Optionen, der sich unter Maßgabe der optimalen Ausübungsstrategie ergibt, von grundlegendem Interesse.

Problematisch im Hinblick auf die praktische Optionsbewertung ist, dass eine analytische Lösung nur für einfache Bewertungsprobleme bzw. Spezialfälle erhältlich ist. Bereits bei amerikanischen Optionen ist man aufgrund der dynamischen Entscheidungsproblematik gezwungen, auf numerisch-approximative Lösungsverfahren zurückgreifen. Auch die häufig eingesetzte Binomialmethode stößt allerdings oft an ihre Grenzen. Dies ist zum einen der Fall, wenn das Underlying keinem geometrischen Brownschen Prozess folgt (Zinsderivate) oder wenn mehrere stochastische Va- riablen (stochastisches Underlying und stochastische Varianz) zu berücksichtigen sind. Zum anderen werden Binomialbäume aber auch schnell sehr aufwändig und unübersichtlich. In diesem Beitrag wird daher ein alternatives Optionsbewertungsverfahren dargestellt. Es handelt sich dabei um ein effizientes und gleichzeitig intuitiv leicht verständliches Verfahren zur Bewertung amerikanischer Optionen, das auf einer von Grant/Vora/Weeks (1997) vorgeschlagenen Vorgehensweise basiert. Es integriert die stochastische Simulation der Zufallsvariablen in den grundsätzlich rekursiv-dynamischen Programmierungsablauf zur Bestimmung der optimalen Ausübungsstrategie.

\section{Das Problem: Bestimmung der optimalen Ausübungsstrategie einer amerikanischen Option}

Im Folgenden wird eine amerikanische Kaufoption auf eine Dividenden zahlende Aktie betrachtet, die innerhalb eines bestimmten Zeitraumes $T$ einmalig zu den diskreten Zeitpunkten $t(t=0,1, \ldots, T)$ ausgeübt werden kann. Der innere Wert $i_{t}$ der Option, der sich bei Ausübung im jeweiligen Zeitpunkt $t$ ergibt, entspricht der positiven Differenz zwischen dem Aktienkurs $V_{t}$ und dem Strike-Preis $I$ :

$i_{t}=\max \left(0, V_{t}-I\right)$

Dabei kennzeichnet $\max (\cdot)$ den Maximumoperator. Begründet ist die Nicht-Negativität des inneren Wertes in der freien Wahl zur Ausübung.

Wird die Ausübung der Option hinausgezögert, so ergibt sich ein Fortführungswert $f_{t}$ :

$f_{t}=E\left(F_{t+1}\right) \cdot e^{-r}$

$E(\cdot)$ beschreibt den Erwartungswertoperator, $F_{t+1}$ den Wert der Option im nächst folgenden potenziellen Ausübungszeitpunkt $t+1$, $e$ die Eulersche Zahl und $r$ den risikolosen Zinssatz. Ausformuliert entspricht der Fortführungswert dem diskontierten Wert der Option, den diese angesichts der stochastischen Aktienkursentwicklung im Zeitpunkt $t+1$ bei optimaler zukünftiger Ausübungsstrategie hat.

Sofortiges Ausüben der Option bedeutet eine Realisation des inneren Wertes und eine gleichzeitige Vernichtung des Fortführungswertes. Ein rational handelnder Entscheider wird deshalb nur dann unverzüglich ausüben, wenn der innere Wert den zu erwartenden Fortführungswert überschreitet. Andernfalls ist Warten vorzuziehen. Der Optionswert $\boldsymbol{F}_{\boldsymbol{t}}$ entspricht dem Maximum aus dem inneren Wert $i_{t}$ und dem Fortführungswert $f_{t}$ : 
$F_{t}=\max \left(i_{t}, f_{t}\right)$

Da in die Berechnung des Fortführungswertes $f_{t}$ der Erwartungswert für die Option im nächst folgenden möglichen Ausübungszeitpunkt $t+1$ einfließt, handelt es sich bei der Bestimmung des Optionswertes $F_{t}$ um ein zeitlich interdependentes oder dynamisches Entscheidungsproblem.

Bei einem hohen Aktienkurs ist der innere Wert $i_{t}$, der sich bei unverzüglicher Ausübung ergibt, höher als der Fortführungswert. In diesem Fall wäre eine unverzügliche Ausübung der Option anzuraten. Unterhalb eines gewissen Aktienkurses ist der Fortführungswert $f_{t}$ höher als der innere Wert $i_{t}$, so dass Warten vorteilhaft ist. In $A b b .1$ (linke Bildhälfte) ist der Funktionsverlauf von innerem Wert $i_{t}$ und Fortführungswert $f_{t}$ in Abhängigkeit vom Kurs $V_{t}$ einer dividendenzahlenden Aktie für einen beliebigen potenziellen Ausübungszeitpunkt $t$ schematisch dargestellt. Bildlich gesprochen sollte die Option bei einem Aktienkurs links des Schnittpunktes zwischen innerem Wert und Fortführungswert nicht unverzüglich ausgeübt werden; rechts davon ist dagegen eine sofortige Ausübung anzuraten. Derjenige Aktienkurs, bei dem sich die Funktionen für den inneren Wert und den Fortführungswert schneiden, wird als kritischer Ausübungswert $V_{t}^{*}$ bezeichnet.

Während sich die linke Darstellung in $A b b .1$ nur auf einen Zeitpunkt bezieht, sind in der rechten Darstellung die kritischen Ausübungswerte $\mathrm{zu}$ verschiedenen potenziellen Ausübungszeitpunkten angezeigt. Jeder der kleinen Sterne entspricht dem für einen potenziellen Ausübungszeitpunkt geltenden kritischen Wert. In ihrer Gesamtheit ergeben die kritischen Werte den sog. kritischen Ausübungspfad. Der kritische Pfad resultiert aus dem unterstellten stochastischen Prozess für das Underlying. Er ist aber unabhängig vom gegenwärtigen Wert des Underlying und definiert die optimale Handlungsstrategie zu den verschiedenen potenziellen Ausübungszeitpunkten. Bildlich gesprochen wird oberhalb des kritischen Pfades die Option sofort ausgeübt, unterhalb wird die weitere Aktienkursentwicklung abgewartet. Charakteristisch für den Verlauf des kritischen Pfa- des ist die exponentielle Abnahme, die Ausdruck der sich mit Abnahme des verbleibenden Ausübungszeitraumes verringernden Flexibilität ist. Da im letzten möglichen Ausübungszeitpunkt $T$ (Verfallszeitpunkt) keine zeitliche Flexibilität mehr vorhanden ist, wird die Kaufoption ausgeübt, wenn der Aktienkurs mindestens dem Strike-Preis entspricht, d.h. wenn der innere Wert positiv ist.

Die größtmögliche Flexibilität hinsichtlich der Modellierung von Unsicherheit weist die stochastische Simulation auf. Beispielsweise können beliebige stochastische Prozesse und multiple stochastische Variablen inkl. ihrer Korrelationen ohne großen Aufwand gleichzeitig über eine Simulation berücksichtigt werden. Das Problem für die Bewertung amerikanischer Optionen besteht zunächst darin, dass der Zeitpfad während des Simulationsvorganges vorwärts durchschritten wird und zum jeweiligen potenziellen Ausübungszeitpunkt unklar ist, ob Halten oder Ausüben die optimale Strategie darstellt. Angesichts der Stärken der stochastischen Simulation wurden verschiedene Vorgehensweisen entwickelt, die dieses Instrument so in einen umfassenden Verfahrensablauf integrieren, dass eine einfach zu handhabende Bewertung komplexer Optionen ermöglicht wird (vgl. z. B. Ibanez/Zapatero, 1998; Grant/ Vora/Weeks, 1997; Dias, 2001). Die Kenntnis dieser Verfahren ist jedoch bisher nicht sehr weit verbreitet. So wurde bis vor kurzem noch vielfach die Auffassung vertreten, dass die Anwendung der stochastischen Simulation auf die Bewertung von Optionen europäischen Typs beschränkt oder für amerikanische Optionen zumindest nicht praktikabel sei (vgl. z. B. Hull, 2000, S. 408; Briys/Bellalah/ Mai/Varenne, 1998, S. 62).

\section{Die begrenzt rekursiv-stochastische Simulation}

Bei der im Folgenden beschriebenen Verfahrenskombination werden die Vorteile der stochastischen Simulation in einem relativ einfachen Ablauf mit den Vorteilen der dynamischen Programmierung kombiniert. Dadurch erhält

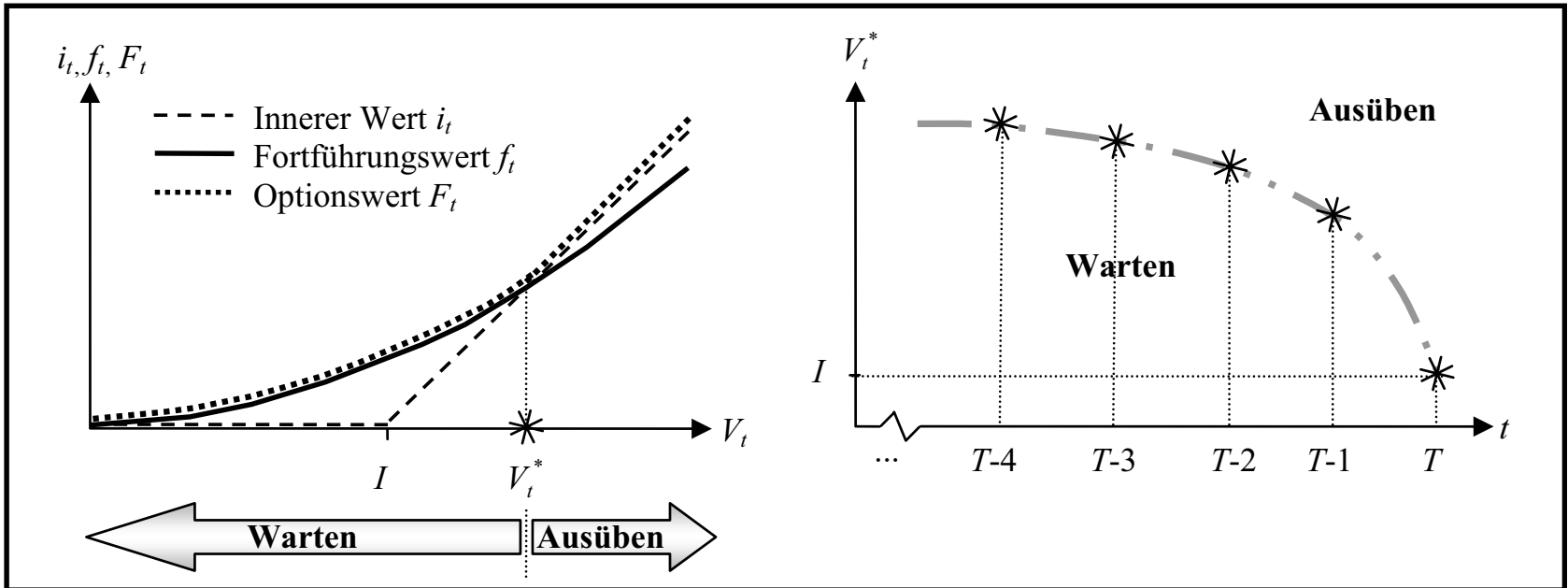

Abb. 1: Innerer Wert vs. Fortführungswert (links; $t<T$ ) und kritischer Ausübungspfad (rechts) 
man größtmögliche Flexibilität zur realistischen Modellierung der Unsicherheit, verbunden mit der Möglichkeit zur Berücksichtigung zeitlicher Flexibilität hinsichtlich der Ausübung der Option im Rahmen eines rückwärts-rekursiven Vorgehens. Das Verfahren kann als „begrenzt rekursiv-stochastische Simulation“ (BRSS) bezeichnet werden.

Der besondere Vorteil der BRSS besteht neben der Einfachheit der Anwendung in ihrer intuitiven Nachvollziehbarkeit: $\mathrm{Zu}$ jedem potenziellen Ausübungszeitpunkt wird der Aktienkurs gesucht, bei dem sich die Handlungsstrategie bzgl. Warten bzw. Ausüben ändert; grafisch geht es also um die Bestimmung des Schnittpunktes zwischen innerem Wert $i_{t}$ und Fortführungswert $f_{t}$ (vgl. $\left.A b b .1\right)$. Im Rahmen der BRSS werden dazu die Ausübungszeitpunkte nacheinander von hinten her betrachtet. Nachdem der kritische Pfad bekannt ist, kann der Optionswert mittels einfacher stochastischer Simulation vorwärts gerichtet bestimmt werden. Kurz zusammengefasst sind folgende Arbeitsschritte erforderlich:

(1) Bestimmung des kritischen Wertes im letzten potenziellen Ausübungszeitpunkt: Ausgangspunkt der rekursiven Bestimmung der Ausübungsstrategie ist der kritische Aktienkurs im letzten potenziellen Ausübungszeitpunkt. Da es zu diesem Zeitpunkt keine Flexibilität mehr gibt, wird die Option ausgeübt, wenn der Aktienkurs den Strike-Preis erreicht und somit einen positiven Rückfluss liefert. Die Kenntnis des kritischen Wertes im letzten potenziellen Ausübungszeitpunkt ist Voraussetzung für die Berechnung des kritischen Wertes im vorletzten möglichen Ausübungszeitpunkt.

(2) Bestimmung des kritischen Wertes im vorletzten potenziellen Ausübungszeitpunkt: Zur Bestimmung des kritischen Wertes werden hypothetische Aktienkurse dahingehend getestet, ob der innere Wert oder der Fortführungswert größer ist. Aufgrund des exponentiell abfallenden Verlaufs des Pfades (vgl. Abb. 1, rechte Bildhälfte) ist klar, dass der bereits bekannte kritische Ausübungswert des jeweils folgenden potenziellen Ausübungszeitpunktes die theoretische Untergrenze (,,begrenzt“) für den kritischen Wert des gerade betrachteten Zeitpunktes darstellt. Für jeden Testaktienkurs lässt sich der innere Wert gemäß (1) berechnen. Der entsprechende Fortführungswert wird mit Hilfe der stochastischen Simulation bestimmt. Dazu werden

\begin{tabular}{|c|c|c|c|}
\hline $\begin{array}{l}\text { gewählter } \\
\text { Testwert }\end{array}$ & $\begin{array}{l}\text { Wnerer } \\
\text { Wert }\end{array}$ & $\begin{array}{l}\text { mittlerer Fortführungswert aus } \\
50.000 \text { Simulationsläufen }\end{array}$ & $\begin{array}{l}\text { Differenz zwischen innerem } \\
\text { Wert und Fortführungswert }\end{array}$ \\
\hline 100 & 0 & 7,5 & $-7,5$ \\
\hline 110 & 10 & 13,5 & $-3,5$ \\
\hline 120 & 20 & 21,0 & $\mathbf{- 1 , 0}$ \\
\hline 130 & 30 & 29,4 & $\mathbf{0 , 6}$ \\
\hline 140 & 40 & 38,4 & 1,6 \\
\hline 150 & 50 & 47,6 & 2,4 \\
\hline
\end{tabular}

Annahmen: Strike-Preis: 100, Zinssatz: $5 \%$ p.a.,

Unsicherheitsvariable: Aktienkurs (geometrisch Brownscher Prozess, ohne Drift, Standardabweichung: $20 \%$ ), Zeitintervall bis Verfall: $1 \mathrm{Jahr}$

Tab. 1: Bestimmung des kritischen Wertes einer Kaufoption im vorletzten potenziellen Ausübungszeitpunkt ausgehend von jedem Testaktienkurs Entwicklungspfade bis zum Verfallszeitpunkt der Option gemäß dem vorgegebenen stochastischen Prozess simuliert. Für jeden Entwicklungspfad wird der Rückfluss der Option im Verfallszeitpunkt berechnet. Der Mittelwert der sich bei den einzelnen Simulationspfaden ergebenden Rückflüsse der Option entspricht dem gesuchten Fortführungswert. Nun werden die beiden Testwerte identifiziert, bei denen die Differenz zwischen dem inneren Wert und dem mittels Simulation bestimmten Fortführungswert das Vorzeichen wechselt. Der zwischen diesen beiden Aktienkursen liegende kritische Ausübungswert wird mittels Interpolation bestimmt. In Tab. 1 ist zur Veranschaulichung der Vorgehensweise ein vereinfachtes numerisches Zahlenbeispiel dargestellt, das aus Gründen der Anschaulichkeit auf ein langes Zeitintervall von einem Jahr abstellt. Nach Interpolation ergibt sich ein kritischer Wert von 126,2 im vorletzten potenziellen Ausübungszeitpunkt. Soll der Interpolationsfehler verringert werden, könnte der Abstand zwischen den Testwerten in einem zweiten Durchlauf verengt werden.

(3) Bestimmung der kritischen Werte zu den übrigen potenziellen Ausübungszeitpunkten: Die unter (2) beschriebene Vorgehensweise findet - dem Grundprinzip der dynamischen Programmierung folgend - rückwärts gerichtet für die Berechnung der kritischen Werte zu allen weiteren potenziellen Ausübungszeitpunkten Anwendung. Allerdings ist bei der simulationsbasierten Bestimmung des Fortführungswertes zu beachten, dass die Option zu mehreren zukünftigen Zeitpunkten ausgeübt werden kann. Dies ist jedoch nicht weiter problematisch, da durch die zuvor angestellten Berechnungen die zukünftige Ausübungsstrategie klar definiert ist.

(4) Bestimmung des relevanten Optionswertes: Nachdem der kritische Ausübungspfad bekannt ist, wird durch eine abschließende Simulation ausgehend vom gegenwärtig beobachtbaren Aktienkurs der Optionswert bestimmt.

\section{Ausblick}

Das hier am Beispiel einer amerikanischen Kaufoption auf eine Aktie dargestellte BRSS-Verfahren, das die dynamische Programmierung mit der stochastischen Simulation verbindet, ist natürlich ohne weiteres auch für die Bewertung amerikanischer Verkaufsoptionen geeignet. Für Aktienkursentwicklungen bildet allerdings der geometrische Brownsche Prozess die Standardannahme, so dass einfache amerikanische Optionen auf Aktien auch mittels Differenzenverfahren (vgl. Brennan/Schwartz, 1977) oder Gitterverfahren (vgl. Cox/Ross/Rubinstein, 1979) bewertet werden können. Die Anwendung der BRSS ist jedoch dann zu empfehlen, wenn bei der Optionsbewertung komplexere stochastische Prozesse oder mehrere Unsicherheitsvariablen berücksichtigt werden müssen und Diffe- 
renzen- bzw. Gitterverfahren nicht mehr gangbar sind. Im Zusammenhang mit Aktienoptionen wird z. B. vielfach die Berücksichtigung einer zeitveränderlichen Volatilität z. B. über einen GARCH-Prozess (vgl. Bollerslev, 1986) diskutiert. Bei der Bewertung von Optionen auf Zinsentwicklungen oder von realen Optionen auf Investitionsrückflüsse (vgl. Dixit/Pindyck, 1994) sind Mean-Reverting-Prozesse oder noch komplexere ARIMA-Prozesse zu modellieren.

Die BRSS ist ein sehr intuitives und zugleich auf eine Vielzahl von Fragestellungen anwendbares Bewertungsverfahren. Oftmals ist es auch von Vorteil, die stochastische Simulation in Kombination mit genetischen Algorithmen als heuristisches Optimierungsverfahren zur Bestimmung der optimalen Ausübungsstrategie und zur Bewertung von Optionen einzusetzen. Dies gilt immer dann, wenn das Bewertungsproblem besonders komplex ist.

Mußhoff/Hirschauer (2003) legen dar, wie verschiedenste numerische Optionsbewertungsverfahren in MS-EXCEL Schritt für Schritt umgesetzt werden können. Darüber hinaus weisen sie darauf hin, welche Verfahren für welche Art der Problemstellung erforderlich sind, ganz nach dem Grundsatz: „So flexibel wie nötig und so einfach wie möglich“.

\section{Literatur}

Zur Einführung in die Optionsbewertung:

Boyle, P.P., A Monte Carlo Approach to Options, in: Journal of Financial Economics, Vol. 4 (1977), S. 323-338.

Brennan, M.J., E.S. Schwartz, The Valuation of American Put Options, in: Journal of Finance, Vol. 32, (1977), S. 449-462.

Briys, E., M. Bellalah, H.M. Mai, F. Varenne, Options, Futures, and Exotic Derivatives, Chichester 1998.

Cox, J.C., S.A. Ross, M. Rubinstein, Option Pricing. A Simplified Approach, in: Journal of Financial Economics, Vol. 7 (1979), S. 229-264.
Dixit, A.K., R.S. Pindyck, Investment under Uncertainty, Princeton 1994.

Haug, E.G., The Complete Guide to Option Pricing Formulas, New York 1998.

Hull, J.C., Options, Futures, and Other Derivatives, 4. Aufl., Toronto 2000 .

Zur Modellierung von stochastischen Prozessen:

Bollerslev, T., A Generalized Autoregressive Conditional Heteroskedasticity, in: Journal of Econometrics, Vol. 31 (1986), S. 307-327.

Box, G.E.P., G.M. Jenkins, Time Series Analysis. Forecasting and Control, San Francisco, 1976.

Pindyck, R.S., D.L. Rubinfeld, Econometric Models and Economic Forecasts, 4. Aufl., Singapore 1998.

$\mathrm{Zu}$ ausgewählten neueren numerischen Optionsbewertungsverfahren:

Dias, M.A.G., Selection of Alternatives of Investment in Information for Oilfield Development Using Evolutionary Real Options Approach with Monte Carlo Simulation, in: Working Paper (2001), PUC-Rio (also presented at the $5^{\text {th }}$ Annual International Conference on Real Options, UCLA, July 2001).

Grant, D., G. Vora, D. Weeks, Simulation and the Early-Exercise Option Problem, in: Journal of Financial Engineering 3, Vol. 5 (1997), S. 211-227.

Ibanez, A., F. Zapatero, Monte Carlo Valuation of American Options through Computation of the Optimal Exercise Frontier, in: Working Paper (1998), Finance and Business Economics Department, University of Southern California, Southern California.

Sowohl eine Einführung in die Optionspreistheorie inkl. kurzer Darstellung stochastischer Prozesse als auch eine Beschreibung der MS-EXCEL-technischen Umsetzung simulationsbasierter Optionsbewertungsverfahren findet sich in:

Mußhoff, O., N. Hirschauer, Bewertung komplexer Optionen. Umsetzung numerischer Verfahren mittels MS-EXCEL und Anwendungsmöglichkeiten der Optionspreistheorie auf Sachinvestitionen (mit CD-ROM), Heidenau 2003.

\section{WiSt Vorschau auf Heft 12/2004}

Prof. Dr. Michael Adams, Das Ende der Mitbestimmung $\bullet$ Prof. Dr. Stefan Eckert, Grounded Theory als methodische Konzeption für wirtschaftswissenschaftliche Forschungsprojekte • Dr. Andreas Größler und Dr. Jörn-Henrik Thun, Die Diffusion von Netzwerkgütern • Prof. Dr. Stephan Paul und Dr. Andreas Horsch, Evolutorische Ökonomik und Lehre von den Unternehmerfunktionen • PD Dr. Bernd Kempa, Geldpolitische Konsequenzen neuerer Entwicklungen im bargeldlosen Zahlungsverkehr $\bullet$ Nikolai Jarre und Prof. Dr. Ulrich van Suntum, John Stuart Mill und der Utilitarismus • Dr. Sebastian Schneider, Einfluss der Geldpolitik auf Investitionsnachfrage und Wirtschaftswachstum • Prof. Dr. Walter Kortmann, Vorteilhafte Eigenschaften kurzfristiger Konkurrenzmarktgleichgewichte $\bullet$ Prof. Dr. Anton Burger und Dipl.Kfm. Philipp Ulbrich, Kapitalmarktorientierung der Rechnungslegung • Dr. Dirk Lepelmeier, Master KAG und Global Custody • Dr. Christian Conrad, Co-opetition und funktionsfähiger Wettbewerb • Prof. Dr. Walter Paul, Die Forderung einer fachübergreifenden betriebswirtschaftlichen Hochschulausbildung vor dem Hintergrund der Bildungs- und Innovationsoffensive - Prof. Dr. Margit Enke und Dipl.-Kffr. Brit Arnold, Kennzahlen des Handels für Platzierungs- und Kalkulationsentscheidungen 